Analele Universităţii de Vest, Timişoara

Seria Matematică - Informatică

LIII, 1, (2015), 137- 150

\title{
Quarter-symmetric metric connection in a $P$-Sasakian manifold
}

\author{
Krishanu Mandal and Uday Chand De
}

\begin{abstract}
In this paper, we consider a quarter-symmetric metric connection in a $P$-Sasakian manifold. We investigate the curvature tensor and the Ricci tensor of a $P$-Sasakian manifold with respect to the quarter-symmetric metric connection. We consider semisymmetric $P$-Sasakian manifold with respect to the quartersymmetric metric connection. Furthermore, we consider generalized recurrent $P$-Sasakian manifolds and prove the non-existence of recurrent and pseudosymmetric $P$-Sasakian manifolds with respect to the quarter-symmetric metric connection. Finally, we construct an example of a 5 -dimensional $P$-Sasakian manifold admitting quarter-symmetric metric connection which verifies Theorem 4.1.
\end{abstract}

AMS Subject Classification (2000). 53C35,53D40.

Keywords. Quarter-symmetric metric connection, P-Sasakian manifold, semi-symmetric manifold, generalized recurrent manifold, recurrent manifold, pseudosymmetric manifold.

\section{Introduction}

A linear connection $\widetilde{\nabla}$ in a Riemannian manifold $M$ is said to be a quartersymmetric connection [10] if the torsion tensor $T$ of the connection $\widetilde{\nabla}$

$$
T(X, Y)=\widetilde{\nabla}_{X} Y-\widetilde{\nabla}_{Y} X-[X, Y]
$$


satisfies

$$
T(X, Y)=\eta(Y) \phi X-\eta(X) \phi Y,
$$

where $\eta$ is a 1 -form and $\phi$ is a $(1,1)$ tensor field. If moreover, a quartersymmetric connection $\widetilde{\nabla}$ satisfies the condition

$$
\left(\widetilde{\nabla}_{X} g\right)(Y, Z)=0
$$

where $X, Y, Z \in \chi(M)$, then $\widetilde{\nabla}$ is said to be a quarter-symmetric metric connection, otherwise it is said to be a quarter-symmetric non-metric connection. If we put $\phi X=X$, then the quarter-symmetric metric connection reduces to a semi-symmetric metric connection [22]. Thus the notion of quartersymmetric connection generalizes the idea of the semi-symmetric connection.

A non-flat $n$-dimensional Riemannian manifold $(n>3)$ is called generalized recurrent [7] if its curvature tensor $R$ satisfies the condition

$$
\left(\nabla_{X} R\right)(Y, Z) W=\alpha(X) R(Y, Z) W+\beta(X)[g(Z, W) Y-g(Y, W) Z],
$$

where $\nabla$ is the Levi-Civita connection and $\alpha$ and $\beta$ are two 1 -forms, $\beta \neq 0$. If $\beta=0$ and $\alpha \neq 0$, then $M$ is called recurrent [21].

A non-flat $n$-dimensional Riemannian manifold $M(n>3)$ is said to be pseudosymmetric [4] if there exists a non-zero 1-form $\alpha$ on $M$ such that

$$
\begin{aligned}
\left(\nabla_{X} R\right)(Y, Z) W= & 2 \alpha(X) R(Y, Z) W+\alpha(Y) R(X, Z) W \\
& +\alpha(Z) R(Y, X) W+\alpha(W) R(Y, Z) X \\
& +g(R(Y, Z) W, X) \rho,
\end{aligned}
$$

where $X, Y, Z, W \in \chi(M)$ and $\rho$ is the corresponding vector field metrically equivalent to the 1 -form $\alpha$ defined by

$$
g(X, \rho)=\alpha(X)
$$

for all $X \in \chi(M)$.

A Riemannian manifold $(M, g)$ is called locally symmetric if its curvature tensor $R$ is parallel, that is, $\nabla R=0$. The notion of semisymmetric, a proper generalization of locally symmetric manifold, is defined by $R(X, Y) \cdot R=0$, where $R(X, Y)$ acts on $R$ as a derivation. A complete intrinsic classification of these manifolds was given by Szabó in [20].

Quarter-symmetric metric connection in a Riemannian manifold studied by several authors such as S.C. Rastogi ([15], [16]), Yano and Imai [23], 
Mukhopadhyay, Roy and Barua [13], Biswas and De [3], De and Mondal [5], Sular et al [19], Kumar et al [11] and many others.

Motivated by the above studies in the present paper, we study quartersymmetric metric connection in a $P$-Sasakian manifold. The paper is organized as follows: In Section 2, we first give a brief account of $P$-Sasakian manifolds. In Section 3, we obtain the expressions of the curvature tensor and the Ricci tensor of a $P$-Sasakian manifold with respect to the quartersymmetric metric connection. Section 4 is devoted to study semisymmetric $P$-sasakian manifolds with respect to the quarter-symmetric metric connection and in this case we have shown that such manifolds are Einstein manifolds with respect to the quarter-symmetric metric connection. Section 5 deals with generalized recurrent $P$-Sasakian manifold with respect to the quarter-symmetric metric connection. In Section 6, we consider pseudosymmetric P-Sasakian manifold with respect to the quarter-symmetric metric connection and we obtain the non-existence of these type of manifolds. Finally, we construct an example of a 5-dimensional $P$-Sasakian manifold admitting quarter-symmetric metric connection which verifies Theorem 4.1.

\section{$2 \quad P$-Sasakian manifolds}

Let $M$ be an $n$-dimensional differentiable manifold of class $C^{\infty}$ in which there are given a $(1,1)$-type tensor field $\phi$, a characteristic vector field $\xi$ and a 1 -form $\eta$ such that

$$
\phi^{2} X=X-\eta(X) \xi, \phi \xi=0, \eta(\xi)=1, \eta(\phi X)=0
$$

Then $(\phi, \xi, \eta)$ is called an almost paracontact structure and $M$ an almost paracontact manifold. Moreover, if $M$ admits a Riemannian metric $g$ such that

$$
g(\xi, X)=\eta(X), g(\phi X, \phi Y)=g(X, Y)-\eta(X) \eta(Y),
$$

then $(\phi, \xi, \eta, g)$ is called an almost paracontact metric structure and $M$ an almost paracontact metric manifold [17]. If $(\phi, \xi, \eta, g)$ satisfy the following equations:

$$
\begin{aligned}
& d \eta=0, \nabla_{X} \xi=\phi X \\
& \left(\nabla_{X} \phi\right) Y=-g(X, Y) \xi-\eta(Y) X+2 \eta(X) \eta(Y) \xi
\end{aligned}
$$


then $M$ is called a para-Sasakian manifold or briefly a $P$-Sasakian manifold [1]. Especially, a $P$-Sasakian manifold $M$ is called a special para-Sasakian manifold or briefly a $S P$-Sasakian manifold [18] if $M$ admits a 1-form $\eta$ satisfying

$$
\left(\nabla_{X} \eta\right)(Y)=-g(X, Y)+\eta(X) \eta(Y) .
$$

Also in a $P$-Sasakian manifold the following relations hold [1], [6], [14]:

$$
\begin{gathered}
S(X, \xi)=-(n-1) \eta(X), Q \xi=-(n-1) \xi \\
\eta(R(X, Y) Z)=g(X, Z) \eta(Y)-g(Y, Z) \eta(X), \\
R(X, \xi) Y=g(X, Y) \xi-\eta(Y) X, \\
R(X, Y) \xi=\eta(X) Y-\eta(Y) X, \\
S(\phi X, \phi Y)=S(X, Y)+(n-1) \eta(X) \eta(Y), \\
\eta(R(X, Y) \xi)=0,
\end{gathered}
$$

for any vector fields $X, Y, Z \in \chi(M)$, where $R$ is the Riemannian curvature tensor, $S$ is the Ricci tensor and $Q$ is the Ricci operator defined by

$$
g(Q X, Y)=S(X, Y) .
$$

$P$-Sasakian manifolds have been studied by several authors such as De et al [8], Yildiz et al [24], Deshmukh and Ahmed [9], Matsumoto, Ianus and Mihai [12], Özgür [14], Adati and Miyazawa [2] and many others.

An almost paracontact Riemannian manifold $M$ is said to be an $\eta$-Einstein manifold if the Ricci tensor $S$ satisfies the condition

$$
S(X, Y)=a g(X, Y)+b \eta(X) \eta(Y)
$$

where $a$ and $b$ are smooth functions on the manifold. In particular, if $b=0$, then $M$ is an Einstein manifold.

\section{Curvature tensor of a $P$-Sasakian manifold with re- spect to the quarter-symmetric metric connection}

Let $\widetilde{\nabla}$ be a linear connection and $\nabla$ be the Levi-Civita connection of a $P$ Sasakian manifold $M$ such that

$$
\widetilde{\nabla}_{X} Y=\nabla_{X} Y+U(X, Y)
$$


where $U$ is a $(1,1)$-type tensor. For $\widetilde{\nabla}$ to be a quarter-symmetric metric connection in $M$, we have [10],

$$
U(X, Y)=\frac{1}{2}\left[T(X, Y)+T^{\prime}(X, Y)+T^{\prime}(Y, X)\right],
$$

where

$$
g\left(T^{\prime}(X, Y), Z\right)=g(T(Z, X), Y)
$$

From (1.2) and (3.3) we get

$$
T^{\prime}(X, Y)=\eta(X) \phi Y-g(\phi X, Y) \xi .
$$

Using (1.2) and (3.4) in (3.2), we have

$$
U(X, Y)=\eta(Y) \phi X-g(\phi X, Y) \xi .
$$

Therefore a quarter-symmetric metric connection $\widetilde{\nabla}$ in a $P$-Sasakian manifold is given by

$$
\widetilde{\nabla}_{X} Y=\nabla_{X} Y+\eta(Y) \phi X-g(\phi X, Y) \xi .
$$

Let $\widetilde{R}$ and $R$ be the curvature tensors with respect to the quarter-symmetric metric connection $\widetilde{\nabla}$ and the Levi-Civita connection $\nabla$ respectively. Then we have from (3.6),

$$
\begin{aligned}
\widetilde{R}(X, Y) U= & R(X, Y) U+3 g(\phi X, U) \phi Y-3 g(\phi Y, U) \phi X \\
& +\eta(U)[\eta(X) Y-\eta(Y) X] \\
& -[\eta(X) g(Y, U)-\eta(Y) g(X, U)] \xi
\end{aligned}
$$

where

$$
\widetilde{R}(X, Y) U=\widetilde{\nabla}_{X} \widetilde{\nabla}_{Y} U-\widetilde{\nabla}_{Y} \widetilde{\nabla}_{X} U-\widetilde{\nabla}_{[X, Y]} U
$$

and $X, Y, Z \in \chi(M)$. By suitable contraction we have from (3.7)

$$
\widetilde{S}(Y, U)=S(Y, U)+2 g(Y, U)-(n+1) \eta(Y) \eta(U)-3 \operatorname{trace} \phi g(\phi Y, U),
$$

where $\widetilde{S}$ and $S$ are the Ricci tensors of the connections $\widetilde{\nabla}$ and $\nabla$, respectively. Hence we have the following theorem:

Theorem 3.1. For a P-Sasakian manifold $(M, g)$ with respect to the quartersymmetric metric connection $\widetilde{\nabla}$

(a) The curvature tensor $\widetilde{R}$ is given by (3.7),

(b) The Ricci tensor $\widetilde{S}$ is symmetric,

(c) $\widetilde{R}(X, Y, Z, W)+\widetilde{R}(X, Y, W, Z)=0$, 
(d) $\widetilde{R}(X, Y, Z, W)+\widetilde{R}(Y, X, Z, W)=0$,

(e) $\widetilde{R}(X, Y, Z, W)=\widetilde{R}(Z, W, X, Y)$,

(f) $\widetilde{S}(Y, \xi)=-2(n-1) \eta(Y)$,

where $X, Y, Z, W \in \chi(M)$.

With the help of (2.7), (2.8) and (2.1) in (3.7) we obtain

$$
\widetilde{R}(\xi, Y) U=2[\eta(U) Y-g(U, Y) \xi]
$$

and

$$
\widetilde{R}(X, Y) \xi=2[\eta(X) Y-\eta(Y) X],
$$

where $X, Y \in \chi(M)$.

\section{Semisymmetric $P$-Sasakian manifolds with respect to the quarter-symmetric metric connection}

In this section we characterize semisymmetric $P$-Sasakian manifolds with respect to the quarter-symmetric metric connection, that is, the curvature tensor satisfies the condition

$$
(\widetilde{R}(\xi, Y) \cdot \widetilde{R})(U, V) W=0 .
$$

This implies

$$
\begin{aligned}
& \widetilde{R}(\xi, Y) \widetilde{R}(U, V) W-\widetilde{R}(\widetilde{R}(\xi, Y) U, V) W \\
& -\widetilde{R}(U, \widetilde{R}(\xi, Y) V) W-\widetilde{R}(U, V) \widetilde{R}(\xi, Y) W=0 .
\end{aligned}
$$

Using (3.9) and (4.1) yields

$$
\begin{aligned}
& 2 \eta(\widetilde{R}(U, V) W) Y-2 g(Y, \widetilde{R}(U, V) W) \xi-2 \eta(U) \widetilde{R}(Y, V) W \\
& +2 g(Y, U) \widetilde{R}(\xi, V) W-2 \eta(V) \widetilde{R}(U, Y) W+2 g(V, Y) \widetilde{R}(U, \xi) W \\
& -2 \eta(W) \widetilde{R}(U, V) Y+2 g(Y, W) \widetilde{R}(U, V) \xi=0 .
\end{aligned}
$$

Taking inner product of (4.2) with $\xi$, we obtain

$$
\begin{aligned}
& 2 \eta(\widetilde{R}(U, V) W) \eta(Y)-2 g(Y, \widetilde{R}(U, V) W)-2 \eta(U) \eta(\widetilde{R}(Y, V) W) \\
& +2 g(Y, U) \eta(\widetilde{R}(\xi, V) W)-2 \eta(V) \eta(\widetilde{R}(U, Y) W)+2 g(V, Y) \eta(\widetilde{R}(U, \xi) W) \\
& -2 \eta(W) \eta(\widetilde{R}(U, V) Y)+2 g(Y, W) \eta(\widetilde{R}(U, V) \xi)=0 .
\end{aligned}
$$


With the help of (3.7), (3.9), (3.10) we get from (4.3)

$$
\begin{aligned}
& 4 \eta(Y)[g(U, W) \eta(V)-g(V, W) \eta(U)]-2\{g(R(U, V) W, Y) \\
& +3 g(\phi U, W) g(\phi V, Y)-3 g(\phi V, W) g(\phi U, Y)+\eta(W)[\eta(U) g(V, Y) \\
& -\eta(V) g(U, Y)]-\eta(Y)[\eta(U) g(V, W)-\eta(V) g(U, W)]\} \\
& -4 \eta(U)[g(Y, W) \eta(V)-g(V, W) \eta(Y)]+4 g(Y, U)[\eta(V) \eta(W) \\
& -g(V, W)]-4 \eta(V)[g(U, W) \eta(Y)-g(Y, W) \eta(U)] \\
& +4 g(V, Y)[g(U, W)-\eta(W) \eta(U)] \\
& -4 \eta(W)[g(U, Y) \eta(V)-g(V, Y) \eta(U)]=0 .
\end{aligned}
$$

Equation (4.4) implies

$$
\begin{aligned}
& g(R(U, V) W, Y)+3 g(\phi U, W) g(\phi V, Y)-3 g(\phi V, W) g(\phi U, Y) \\
& +g(V, Y) \eta(U) \eta(W)-g(U, Y) \eta(V) \eta(W)-g(V, W) \eta(U) \eta(Y) \\
& +g(U, W) \eta(V) \eta(Y)+2 g(Y, U) g(V, W)-2 g(U, W) g(V, Y)=0 .
\end{aligned}
$$

Now putting $V=W=e_{i}$ in (4.5), where $\left\{e_{i}\right\}$ is an orthonormal basis of the tangent space at each point of the manifold, and taking summation over $i=1,2, \ldots, n$, we get

$$
S(U, Y)=-2 n g(U, Y)+(n+1) \eta(U) \eta(Y)+3 \operatorname{trace} \phi g(\phi U, Y) .
$$

Again from (3.8) and (4.6) we have

$$
\widetilde{S}(U, Y)=-2(n-1) g(U, Y) .
$$

By making contraction of (4.7) we obtain

$$
\widetilde{r}=-2 n(n-1) .
$$

This leads to the following theorem:

Theorem 4.1. If a P-Sasakian manifold is semisymmetric with respect to the quarter-symmetric metric connection, then the manifold is an Einstein manifold with respect to the quarter-symmetric metric connection and the scalar curvature with respect to the quarter-symmetric metric connection is a negative constant.

\section{Generalized recurrent $P$-Sasakian manifolds with re- spect to the quarter-symmetric metric connection}

In this section we consider generalized recurrent $P$-Sasakian manifolds with respect to the quarter-symmetric metric connection $\widetilde{\nabla}$. Let us assume that 
there exists a generalized recurrent $P$-Sasakian manifold $M$ with respect to the quarter-symmetric metric connection $\widetilde{\nabla}$. Then from (1.4), we have

$$
\left(\widetilde{\nabla}_{X} \widetilde{R}\right)(Y, Z) W=\alpha(X) \widetilde{R}(Y, Z) W+\beta(X)[g(Z, W) Y-g(Y, W) Z],
$$

for all vector fields $X, Y, Z, W \in \chi(M)$. Substituting $Y=W=\xi$ in (5.1) we obtain

$$
\left(\widetilde{\nabla}_{X} \widetilde{R}\right)(\xi, Z) \xi=\alpha(X) \widetilde{R}(\xi, Z) \xi+\beta(X)[\eta(Z) \xi-Z] .
$$

We have from (3.10)

$$
\left(\widetilde{\nabla}_{X} \widetilde{R}\right)(Y, Z) \xi=2\left[\left(\left(\widetilde{\nabla}_{X} \eta\right) Y\right) Z-\left(\left(\widetilde{\nabla}_{X} \eta\right) Z\right) Y\right] .
$$

On the other hand using (3.6), (2.3) and (1.3) we get

$$
\left(\widetilde{\nabla}_{X} \eta\right) Y=2 g(Y, \phi X) \text {. }
$$

So by the use of (5.4) in (5.3) we have

$$
\left(\widetilde{\nabla}_{X} \widetilde{R}\right)(Y, Z) \xi=4[g(Y, \phi X) Z-g(Z, \phi X) Y] .
$$

Putting $Y=\xi$ in the above equation yields

$$
\left(\widetilde{\nabla}_{X} \widetilde{R}\right)(\xi, Z) \xi=-4 g(Z, \phi X) \xi
$$

Again from (3.10) we have

$$
\widetilde{R}(\xi, Z) \xi=2[Z-\eta(Z) \xi] .
$$

Thus we get from (5.2) and (5.7)

$$
\left(\widetilde{\nabla}_{X} \widetilde{R}\right)(\xi, Z) \xi=2 \alpha(X)[Z-\eta(Z) \xi]+\beta(X)[\eta(Z) \xi-Z] .
$$

Hence comparing the right hand sides of the equations (5.6) and (5.8) we obtain

$$
-4 g(Z, \phi X) \xi=2 \alpha(X)[Z-\eta(Z) \xi]-\beta(X)[Z-\eta(Z) \xi] .
$$

Operating $\phi$ both sides of (5.9) and using (2.1), we get

$$
\beta(X)=2 \alpha(X) .
$$

This leads to the following theorem:

Theorem 5.1. If a P-Sasakian manifold is generalized recurrent with respect to the quarter-symmetric metric connection $\widetilde{\nabla}$, then the associated 1-forms are related by $\beta=2 \alpha$. 
Next, we consider recurrent $P$-Sasakian manifold $M$ with respect to the quarter-symmetric metric connection $\widetilde{\nabla}$. Then from (1.4), we have

$$
\left(\widetilde{\nabla}_{X} \widetilde{R}\right)(Y, Z) W=\alpha(X) \widetilde{R}(Y, Z) W
$$

for all vector fields $X, Y, Z, W \in \chi(M)$. From Theorem 5.1. we obtain

$$
\alpha(X)=0 .
$$

Hence we have the following corollary:

Corollary 5.2. There is no recurrent P-Sasakian manifold with respect to the quarter-symmetric metric connection $\widetilde{\nabla}$.

\section{Pseudosymmetric $P$-Sasakian manifolds with respect to the quarter-symmetric metric connection}

This section is devoted to study of pseudosymmetric $P$-Sasakian manifolds with respect to the quarter-symmetric metric connection. We prove the following theorem:

Theorem 6.1. There is no pseudosymmetric P-Sasakian manifold with respect to the quarter-symmetric metric connection $\widetilde{\nabla}$.

Proof. Let us assume that there exists a pseudosymmetric P-Sasakian manifold with respect to the quarter-symmetric metric connection $\widetilde{\nabla}$. Then we get from (1.5)

$$
\begin{aligned}
\left(\widetilde{\nabla}_{X} \widetilde{R}\right)(Y, Z) W= & 2 \alpha(X) \widetilde{R}(Y, Z) W+\alpha(Y) \widetilde{R}(X, Z) W \\
& +\alpha(Z) \widetilde{R}(Y, X) W+\alpha(W) \widetilde{R}(Y, Z) X \\
& +g(\widetilde{R}(Y, Z) W, X) \rho .
\end{aligned}
$$

So contracting $Y$ in (6.1), we have

$$
\begin{aligned}
\left(\widetilde{\nabla}_{X} \widetilde{S}\right)(Z, W)= & 2 \alpha(X) \widetilde{S}(Z, W)+\alpha(\widetilde{R}(X, Z) W) \\
& +\alpha(Z) \widetilde{S}(X, W)+\alpha(W) \widetilde{S}(Z, X) \\
& +g(\widetilde{R}(\rho, Z) W, X) .
\end{aligned}
$$

Substituting $W=\xi$ in (6.2) we get

$$
\begin{aligned}
\left(\widetilde{\nabla}_{X} \widetilde{S}\right)(Z, \xi)= & 2 \alpha(X) \widetilde{S}(Z, \xi)+\alpha(\widetilde{R}(X, Z) \xi) \\
& +\alpha(Z) \widetilde{S}(X, \xi)+\alpha(\xi) \widetilde{S}(Z, X) \\
& +g(\widetilde{R}(\rho, Z) \xi, X) .
\end{aligned}
$$


From Theorem 3.1. we get

$$
\widetilde{S}(Z, \xi)=-2(n-1) \eta(Z) .
$$

Hence using (5.4) it follows that

$$
\left(\widetilde{\nabla}_{X} \widetilde{S}\right)(Z, \xi)=-4(n-1) g(Z, \phi X)
$$

On the other hand, in view of (2.1) and Theorem 3.1. we obtain

$$
\begin{aligned}
\left(\widetilde{\nabla}_{X} \widetilde{S}\right)(Z, \xi)= & -4 n \alpha(X) \eta(Z)+2 \eta(X) \alpha(Z) \\
& -2(n-1) \alpha(Z) \eta(X)+2 \alpha(\xi) g(X, Z) \\
& +\alpha(\xi) \widetilde{S}(X, Z) .
\end{aligned}
$$

From (6.4) and (6.5), we get

$$
\begin{aligned}
-4(n-1) g(Z, \phi X)= & -4 n \alpha(X) \eta(Z)+2 \eta(X) \alpha(Z) \\
& -2(n-1) \alpha(Z) \eta(X)+2 \alpha(\xi) g(X, Z) \\
& +\alpha(\xi) \widetilde{S}(X, Z) .
\end{aligned}
$$

Taking $X=\xi$ in the above equation gives

$$
\begin{aligned}
-4(n-1) g(Z, \phi \xi)= & -4 n \alpha(\xi) \eta(Z)+2 \eta(\xi) \alpha(Z) \\
& -2(n-1) \alpha(Z) \eta(\xi)+2 \alpha(\xi) \eta(Z) \\
& +\alpha(\xi) \widetilde{S}(\xi, Z) .
\end{aligned}
$$

By making use of (2.1), (2.2), (1.6) and Theorem 3.1. in (6.7) yields

$$
(2-3 n) \alpha(\xi) \eta(Z)+(2-n) \alpha(Z)=0 .
$$

Replacing $Z$ with $\xi$ in (6.8), we have (since $n>3$ )

$$
\alpha(\xi)=0
$$

Now using (6.9) it follows from (6.8) that

$$
\alpha(Z)=0,
$$

for every vector field $Z$ on $M$, which implies that $\alpha=0$ on $M$. This contradicts to our assumption.

Thus the proof of our theorem is completed. 


\section{Example of a 5-dimensional $P$-Sasakian manifold ad- mitting quarter-symmetric metric connection}

Example 7.1. We consider the 5-dimensional manifold $\left\{(x, y, z, u, v) \in \mathbb{R}^{5}\right\}$, where $(x, y, z, u, v)$ are the standard coordinates in $\mathbb{R}^{5}$.

We choose the vector fields

$$
e_{1}=\frac{\partial}{\partial x}, e_{2}=e^{-x} \frac{\partial}{\partial y}, e_{3}=e^{-x} \frac{\partial}{\partial z}, e_{4}=e^{-x} \frac{\partial}{\partial u}, e_{5}=e^{-x} \frac{\partial}{\partial v},
$$

which are linearly independent at each point of $M$.

Let $g$ be the Riemannian metric defined by

$$
g\left(e_{i}, e_{j}\right)= \begin{cases}1 & \text { if } \quad i=j \\ 0 & \text { if } \quad i \neq j ; i, j=1,2,3,4,5\end{cases}
$$

Let $\eta$ be the 1-form defined by

$$
\eta(Z)=g\left(Z, e_{1}\right)
$$

for any $Z \in \chi(M)$.

Let $\phi$ be the $(1,1)$-tensor field defined by

$$
\phi\left(e_{1}\right)=0, \phi\left(e_{2}\right)=e_{2}, \phi\left(e_{3}\right)=e_{3}, \phi\left(e_{4}\right)=e_{4}, \phi\left(e_{5}\right)=e_{5} .
$$

Using the linearity of $\phi$ and $g$, we have

$$
\eta\left(e_{1}\right)=1, \phi^{2} Z=Z-\eta(Z) e_{1}
$$

and

$$
g(\phi Z, \phi U)=g(Z, U)-\eta(Z) \eta(U),
$$

for any vector fields $Z, U \in \chi(M)$. Thus for $e_{1}=\xi$, the structure $(\phi, \xi, \eta, g)$ defines an almost paracontact metric structure on $M$.

Then we have

$$
\begin{aligned}
& {\left[e_{1}, e_{2}\right]=-e_{2},\left[e_{1}, e_{3}\right]=-e_{3},\left[e_{1}, e_{4}\right]=-e_{4},\left[e_{1}, e_{5}\right]=-e_{5},} \\
& {\left[e_{2}, e_{3}\right]=\left[e_{2}, e_{4}\right]=\left[e_{2}, e_{5}\right]=\left[e_{3}, e_{4}\right]=\left[e_{3}, e_{5}\right]=\left[e_{4}, e_{5}\right]=0 .}
\end{aligned}
$$

The Levi-Civita connection $\nabla$ of the metric tensor $g$ is given by Koszul's formula which is given by

$$
\begin{aligned}
2 g\left(\nabla_{X} Y, Z\right)= & X g(Y, Z)+Y g(Z, X)-Z g(X, Y) \\
& -g(X,[Y, Z])-g(Y,[X, Z])+g(Z,[X, Y]) .
\end{aligned}
$$


Taking $e_{1}=\xi$ and using (7.1), we get the following:

$$
\begin{aligned}
& \nabla_{e_{1}} e_{1}=0, \nabla_{e_{1}} e_{2}=0, \nabla_{e_{1}} e_{3}=0, \nabla_{e_{1}} e_{4}=0, \nabla_{e_{1}} e_{5}=0, \\
& \nabla_{e_{2}} e_{1}=e_{2}, \nabla_{e_{2}} e_{2}=-e_{1}, \nabla_{e_{2}} e_{3}=0, \nabla_{e_{2}} e_{4}=0, \nabla_{e_{2}} e_{5}=0, \\
& \nabla_{e_{3}} e_{1}=e_{3}, \nabla_{e_{3}} e_{2}=0, \nabla_{e_{3}} e_{3}=-e_{1}, \nabla_{e_{3}} e_{4}=0, \nabla_{e_{3}} e_{5}=0, \\
& \nabla_{e_{4}} e_{1}=e_{4}, \nabla_{e_{4}} e_{2}=0, \nabla_{e_{4}} e_{3}=0, \nabla_{e_{4}} e_{4}=-e_{1}, \nabla_{e_{4}} e_{5}=0, \\
& \nabla_{e_{5}} e_{1}=e_{5}, \nabla_{e_{5}} e_{2}=0, \nabla_{e_{5}} e_{3}=0, \nabla_{e_{5}} e_{4}=0, \nabla_{e_{5}} e_{5}=-e_{1} .
\end{aligned}
$$

Using the above equations in (3.6) yields

$$
\begin{aligned}
& \widetilde{\nabla}_{e_{1}} e_{1}=0, \widetilde{\nabla}_{e_{1}} e_{2}=0, \widetilde{\nabla}_{e_{1}} e_{3}=0, \widetilde{\nabla}_{e_{1}} e_{4}=0, \widetilde{\nabla}_{e_{1}} e_{5}=0, \\
& \widetilde{\nabla}_{e_{2}} e_{1}=2 e_{2}, \widetilde{\nabla}_{e_{2}} e_{2}=-2 e_{1}, \widetilde{\nabla}_{e_{2}} e_{3}=0, \widetilde{\nabla}_{e_{2}} e_{4}=0, \widetilde{\nabla}_{e_{2}} e_{5}=0, \\
& \widetilde{\nabla}_{e_{3}} e_{1}=2 e_{3}, \widetilde{\nabla}_{e_{3}} e_{2}=0, \widetilde{\nabla}_{e_{3}} e_{3}=-2 e_{1}, \widetilde{\nabla}_{e_{3}} e_{4}=0, \widetilde{\nabla}_{e_{3}} e_{5}=0, \\
& \widetilde{\nabla}_{e_{4}} e_{1}=2 e_{4}, \widetilde{\nabla}_{e_{4}} e_{2}=0, \widetilde{\nabla}_{e_{4}} e_{3}=0, \widetilde{\nabla}_{e_{4}} e_{4}=-2 e_{1}, \widetilde{\nabla}_{e_{4}} e_{5}=0, \\
& \widetilde{\nabla}_{e_{5}} e_{1}=2 e_{5}, \widetilde{\nabla}_{e_{5}} e_{2}=0, \widetilde{\nabla}_{e_{5}} e_{3}=0, \widetilde{\nabla}_{e_{5}} e_{4}=0, \widetilde{\nabla}_{e_{5}} e_{5}=-2 e_{1} .
\end{aligned}
$$

By the above results, we can easily obtain the non-vanishing components of the curvature tensors as follows:

$$
\begin{aligned}
& R\left(e_{1}, e_{2}\right) e_{1}=e_{2}, R\left(e_{1}, e_{2}\right) e_{2}=-e_{1}, R\left(e_{1}, e_{3}\right) e_{1}=e_{3}, R\left(e_{1}, e_{3}\right) e_{3}=-e_{1}, \\
& R\left(e_{1}, e_{4}\right) e_{1}=e_{4}, R\left(e_{1}, e_{4}\right) e_{4}=-e_{1}, R\left(e_{1}, e_{5}\right) e_{1}=e_{5}, R\left(e_{1}, e_{5}\right) e_{5}=-e_{1}, \\
& R\left(e_{2}, e_{3}\right) e_{2}=e_{3}, R\left(e_{2}, e_{3}\right) e_{3}=-e_{2}, R\left(e_{2}, e_{4}\right) e_{2}=e_{4}, R\left(e_{2}, e_{4}\right) e_{4}=-e_{2}, \\
& R\left(e_{2}, e_{5}\right) e_{2}=e_{5}, R\left(e_{2}, e_{5}\right) e_{5}=-e_{2}, R\left(e_{3}, e_{4}\right) e_{3}=e_{4}, R\left(e_{3}, e_{4}\right) e_{4}=-e_{3}, \\
& R\left(e_{3}, e_{5}\right) e_{3}=e_{5}, R\left(e_{3}, e_{5}\right) e_{5}=-e_{3}, R\left(e_{4}, e_{5}\right) e_{4}=e_{5}, R\left(e_{4}, e_{5}\right) e_{5}=-e_{4}, \\
& \widetilde{R}\left(e_{1}, e_{2}\right) e_{1}=2 e_{2}, \widetilde{R}\left(e_{1}, e_{2}\right) e_{2}=-2 e_{1}, \widetilde{R}\left(e_{1}, e_{3}\right) e_{1}=2 e_{3}, \\
& \widetilde{R}\left(e_{1}, e_{3}\right) e_{3}=-2 e_{1}, \widetilde{R}\left(e_{1}, e_{4}\right) e_{1}=2 e_{4}, \widetilde{R}\left(e_{1}, e_{4}\right) e_{4}=-2 e_{1}, \\
& \widetilde{R}\left(e_{1}, e_{5}\right) e_{1}=2 e_{5}, \widetilde{R}\left(e_{1}, e_{5}\right) e_{5}=-2 e_{1}, \widetilde{R}\left(e_{2}, e_{3}\right) e_{2}=2 e_{3}, \\
& \widetilde{R}\left(e_{2}, e_{3}\right) e_{3}=-2 e_{2}, \widetilde{R}\left(e_{2}, e_{4}\right) e_{2}=2 e_{4}, \widetilde{R}\left(e_{2}, e_{4}\right) e_{4}=-2 e_{2}, \\
& \widetilde{R}\left(e_{2}, e_{5}\right) e_{2}=2 e_{5}, \widetilde{R}\left(e_{2}, e_{5}\right) e_{5}=-2 e_{2}, \widetilde{R}\left(e_{3}, e_{4}\right) e_{3}=2 e_{4}, \\
& \widetilde{R}\left(e_{3}, e_{4}\right) e_{4}=-2 e_{3}, \widetilde{R}\left(e_{3}, e_{5}\right) e_{3}=2 e_{5}, \widetilde{R}\left(e_{3}, e_{5}\right) e_{5}=-2 e_{3}, \\
& \widetilde{R}\left(e_{4}, e_{5}\right) e_{4}=2 e_{5}, \widetilde{R}\left(e_{4}, e_{5}\right) e_{5}=-2 e_{4} .
\end{aligned}
$$

From the expressions of the curvature tensor it follows that the manifold is a manifold of constant curvature -2 with respect to the quarter-symmetric metric connection. Hence the manifold is semisymmetric with respect to the 
quarter-symmetric metric connection. Using the above expressions of the curvature tensor we get

$$
\widetilde{S}\left(e_{1}, e_{1}\right)=\widetilde{S}\left(e_{2}, e_{2}\right)=\widetilde{S}\left(e_{3}, e_{3}\right)=\widetilde{S}\left(e_{4}, e_{4}\right)=\widetilde{S}\left(e_{5}, e_{5}\right)=-8
$$

Hence the scalar curvature

$$
\widetilde{r}=-40 .
$$

It can be easily verified that the manifold is an Einstein manifold.

Thus Theorem 4.1. is verified.

\section{Acknowledgement}

The authors are thankful to the referee for his valuable comments and suggestions towards the improvement of the paper.

\section{References}

[1] T. Adati and K. Matsumoto, On conformally recurrent and conformally symmetric P-Sasakian manifolds, TRU Math., 13, (1977), 25-32

[2] T. Adati and T. Miyazawa, Some properties of P-Sasakian manifolds, TRU Math., 13, (1977), 33-42

[3] S.C. Biswas and U.C. De, Quarter-symmetric metric connection in $S P$-Sasakian manifold, Common. Fac. Sci. Univ. Ank. Al., 46, (1997), 49-56

[4] M.C. Chaki, On pseudo symmetric manifolds, An. Stiint. Univ. Al. I. Cuza Iasi Sect. I a Mat., 33, (1987), 53-58

[5] U.C. De and A.K. Mondal, Quarter-symmetric metric connection on 3dimensional quasi-Sasakian manifolds, SUT J. of Math., 46, (2010), 35-52

[6] U.C. De and J.C. Ghosh, On a type of contact manifold, Note Mat., 14, (1994), $155-160$

[7] U.C. De and N. Guha, On generalized recurrent manifolds, J. National Academy of Math. India., 9, (1991), 85-92

[8] U.C. De, C. Özgür, K. Arslan, C. Murathan, and A. Yildiz, On a type of P-Sasakian manifolds, Mathematica Balkanica, 22, (2008), 25-36

[9] S. Deshmukh and S. Ahmed, Para Sasakian manifolds isometrically immersed in spaces of constant curvature, Kyungpook J. Math., 20, (1980), 112-121

[10] S. Golab, On semi-symmetric and quarter-symmetric linear connections, Ten$\operatorname{sor}(N . S),. \mathbf{2 9},(1975), 249-254$ 
[11] K.T.P. Kumar, Venkatesha, and C.S. Bagewadi, On $\phi$-Recurrent Para-Sasakian manifold admitting quarter-symmetric metric connection, ISRN Geometry, 2012, (2012), 1-10

[12] K. Matsumoto, S. Ianus, and I. Mihai, On $P$-Sasakian manifolds which admit certain tensor fields, Publ. Math. Debrecen, 33, (1986), 61-65

[13] S. Mukhopadhyay, A.K. Roy, and B. Barua, Some properties of quartersymmetric metric connection on a Riemannian manifold, Soochow J. of Math., 17, (1991), 205-211

[14] C. Özgür, On a class of Para-Sasakian manifolds, Turkish J. Math., 29, (2005), $249-257$

[15] S.C. Rastogi, On quarter-symmetric metric connection, C. R. Acad. Sci. Bulgar, 31, (1978), 811-814

[16] S.C. Rastogi, On quarter-symmetric metric connection, Tensor, N.S., 44, (1987), $133-141$

[17] I. Sató, On a structure similar to the almost contact structure, Tensor (N.S.), 30, (1976), 219-224

[18] I. Sató and K. Matsumoto, On P-Sasakian manifolds satisfying certain conditions, Tensor, N. S., 33, (1979), 173-178

[19] S. Sular, C. Özgür, and U.C. De, Quarter-symmetric metric connection in a Kenmotsu manifold, SUT J. of Math., 44, (2008), 297-306

[20] Z.I. Szabó, Structure theorems on Riemannian spaces satisfying $R(X, Y) \cdot R=0$ I the local version, J. Diff. Geometry, 17, (1982), 531-582

[21] A.G. Walker, On Ruse's spaces of recurrent curvature, Proc. Lon. Math. Soc., 52, (1951), 36-64

[22] K. Yano, On semi-symmetric metric connection, Rev. Roumaine Math. Pures Appl., 15, (1970), 1579-1586

[23] K. Yano and T. Imai, Quarter-symmetric metric connections and their curvature tensors, Tensor (N.S.), 38, (1982), 13-18

[24] A. Yildiz, M. Turan, and B.E. Acet, On Para-Sasakian manifolds, Dumlupinar Üniversitesi, 24, (2011), 27-34

Krishanu Mandal

Department of Pure Mathematics, University of Calcutta, 35, Ballygunge Circular Road, Kolkata- 700019, West Bengal, INDIA

E-mail: krishanu.mandal013@gmail.com

Uday Chand De

Department of Pure Mathematics, University of Calcutta, 35, Ballygunge Circular Road, Kolkata- 700019, West Bengal, INDIA

E-mail: uc_de@yahoo.com

Received: 10.02 .2015

Accepted: 14.03 .2015 